\title{
Monte Carlo calculations of pair production in high-intensity laser-plasma interactions
}

\author{
R Duclous ${ }^{1}$, J G Kirk ${ }^{2}$ \& A R Bell ${ }^{1,3}$ \\ ${ }^{1}$ Clarendon Laboratory, University of Oxford, Parks Road, Oxford UK OX1 3PU \\ 2 Max-Planck-Institut für Kernphysik, Postfach 1039 80, 69029 Heidelberg, Germany \\ ${ }^{3}$ STFC Rutherford Appleton Laboratory, Didcot, Oxfordshire UK OX11 0QX \\ E-mail: r.duclous1@physics.ox.ac.uk
}

\begin{abstract}
.
Gamma-ray and electron-positron pair production will figure prominently in laser-plasma experiments with next generation lasers. Using a Monte Carlo approach we show that straggling effects arising from the finite recoil an electron experiences when it emits a high energy photon, increase the number of pairs produced on further interaction with the laser fields.
\end{abstract}

\section{Introduction}

Next generation lasers such as ELI [1, 2] and VULCAN [3, 4] are expected to achieve intensities of $10^{23} \mathrm{~W} \mathrm{~cm}^{-2}$ or greater, opening up rich opportunities to study QED processes in the strong field regime[5, 6, 7]. One of these processes is pair production in counterpropagating beams, which is a significant effect at $10^{23} \mathrm{~W} \mathrm{~cm}^{-2}[8]$, and, at $10^{24} \mathrm{~W} \mathrm{~cm}^{-2}$ is sufficiently strong that a cascade may develop in which each electron produces a pair that in turn produces further pairs, to initiate an avalanche.

In intense laser beams, the dominant route to pair production is a two-stage process. Firstly, an electron with Lorentz factor $\gamma$ interacts with the laser fields $(\boldsymbol{E}, \boldsymbol{B})$ to produce a gamma-ray with high energy $h v$. Secondly, the gamma-ray interacts with the same fields as it propagates to produce an electron-positron pair [8, 9]. The crucial parameters for the twostage process are (i) $\eta \approx \gamma\left|\boldsymbol{E}_{\perp}+\boldsymbol{v} \times \boldsymbol{B}\right| / E_{\text {cri }}$ t where $E_{\text {crit }}=1.3 \times 10^{18} \mathrm{Vm}^{-1}$ is the Schwinger field, and (ii) $\chi=h v\left|\boldsymbol{E}_{\perp}+(c \boldsymbol{k} / k) \times \boldsymbol{B}\right| / 2 m_{\mathrm{e}} c^{2} E_{\text {crit }}$ where $\hbar \boldsymbol{k}$ is the gamma-ray momentum and $\boldsymbol{E}_{\perp}$ is the component of the electric field perpendicular to the direction of motion of the electron or photon, as appropriate. Strong field QED effects such as pair production become important when these parameters reach unity. The energy of the gamma-ray photon is typically $h v \approx 0.44 \eta \gamma m c^{2}$, implying $\chi \approx 0.22 \eta^{2}$, and the cross-section for pair production is proportional to $\exp (-4 / 3 \chi)$ for $\chi \ll 1$. Because of the exponential cut-off to the crosssection, the process is very sensitive to the value of $\eta$ reached by the electron, when the regime of strong QED is approached. This leads to an abrupt laser-intensity threshold for

$\ddagger$ This expression is generally accurate for $\gamma \gg 1$, a precise definition is given in (2) 
pair production as noted by [8], [9] and [10], where a small increase in $\eta$ can lead to a large increase in pair production.

In [8], [9] and [11], a semi-classical treatment was used in which electrons were subject to a continuous loss of energy through radiation of gamma-rays. In this continuous model, the radiation reaction force is approximated, to the lowest order in $1 / \gamma$, by the expression

$$
\mathbf{f}_{\text {rad }}=-\frac{2}{3} \alpha_{f} \frac{m_{\mathrm{e}}^{2} c^{3}}{\hbar} \eta^{2} g(\eta) \frac{\mathbf{p}}{p} \quad, \quad g(\eta)=\frac{3 \sqrt{3}}{2 \pi \eta^{2}} \int_{0}^{\infty} F(\eta, \chi) d \chi \quad,
$$

where $g(\eta) \in[0,1]$ accounts for the reduction of the total power radiated by the electron. $F(\eta, \chi)$ is the quantum synchrotron emissivity, as defined in Appendix A of [9]. However, in reality, gamma-ray emission is a random quantum process [12, 13]. As $\eta$ approaches unity, the energy of the emitted gamma-ray becomes a substantial fraction, typically $0.44 \eta$, of the kinetic energy of the electron before emission, and the resulting electron trajectory starts to fluctuate substantially away from the semi-classical average. This enhances pair production in two ways. Firstly, if an accelerating electron propagates by chance over an unusually large distance before emitting a gamma-ray, it may reach a large Lorentz factor $\gamma$ and emit a gamma-ray with an unusually high energy. This, in turn, has a much increased probability of producing a pair, as compared to the photons emitted by an electron moving on a classical trajectory in the same field. Such events cumulate, and we show in 3.1 that this straggling effect [12] enhances pair production. Secondly, the deviations in the trajectory caused by straggling lead the electron to sample the laser fields at locations other than those on the classical path. In [9], it was shown that, under the influence of continuous radiation losses, electrons naturally migrate towards points in the laser field at which they emit few high-energy gamma-rays. In the case of counter-propagating circularly polarised waves, these are the nodes in the electric field. Once an electron settles at an $\boldsymbol{E}=0$ node, its Lorentz factor drops and pair production ceases. In contrast, we show in 3.2 that straggling causes electrons to migrate more slowly towards these nodes. Consequently, electrons undergoing discontinuous momentum changes retain a large Lorentz factor for longer than their semi-classical counterparts, and emit a larger number of high energy gamma-rays, which subsequently produce more pairs.

In this paper, we extend the calculations of [8] and [9] by modelling radiation loss as a discontinuous process that leads to straggling effects on the electron trajectory. This is done using a Monte-Carlo method that is described in section 2. In section 3, we compare the continuous with the more realistic discontinuous loss case, and show that the latter produces a larger number of pairs at laser intensities around $10^{23} \mathrm{~W} \mathrm{~cm}^{-2}$. Pair production at $10^{24} \mathrm{~W} \mathrm{~cm}^{-2}$ is relatively little affected by straggling since, at this intensity, even semi-classical, continuous electron trajectories lead to gamma-rays that are well over the threshold for pair production.

\section{Description of the Monte Carlo algorithm}

In 4-vector notation, the quantities $\eta$ and $\chi$ are defined as

$$
\eta=\frac{e \hbar}{m_{\mathrm{e}}^{3} c^{4}}\left|F_{\mu \nu} p^{\nu}\right| \quad, \quad \chi=\frac{e \hbar^{2}}{2 m_{\mathrm{e}}^{3} c^{4}}\left|F_{\mu \nu} k^{v}\right|,
$$


for an electron and photon whose four-momenta are $p^{v}$ and $\hbar k^{v}$, respectively, with $F_{\mu \nu}$ the electromagnetic field tensor. In the following, we make use of the approach presented in [9], that retains the weak, quasi-stationary field approximation. The probabilities for gamma-ray photon emission and the subsequent pair production are in this approximation functions of $\eta$ and $\chi$ only. An equivalent system is then chosen, for the same given $\chi$ and $\eta$, in which the electron moves in a plane perpendicular to a uniform, static $B$ field. This allows us to use the transition probabilities as formulated in [14].

The radiation emitted by the particle due to its acceleration in the laser fields - here called synchrotron radiation — is assumed to be a random walk process [12, 13]. The probability of emission of a gamma-ray photon is governed by an optical depth $\tau_{\mathrm{e}}$. At the start of the calculation, and immediately following the emission of a photon, the current optical depth $\tau_{\mathrm{e}}$ is set to zero and a randomly chosen 'final' optical depth $\tau_{\mathrm{e}}^{(\mathrm{f})}$ is assigned to the electron. The current optical depth $\tau_{\mathrm{e}}$ increases as the electron propagates until it reaches $\tau_{\mathrm{e}}^{(\mathrm{f})}$, at which point a photon is emitted. At each computational timestep the electron's position, momentum and current optical depth are updated according to the equations

$$
\begin{aligned}
\frac{\mathrm{d} \boldsymbol{p}}{\mathrm{d} t} & =-e(\boldsymbol{E}+\boldsymbol{v} \wedge \boldsymbol{B}), \\
\frac{\mathrm{d} \boldsymbol{x}}{\mathrm{d} t} & =\frac{\boldsymbol{p}}{m_{\mathrm{e}} \gamma}, \\
\frac{\mathrm{d} \tau_{\mathrm{e}}}{\mathrm{d} t} & =\int_{0}^{\eta / 2} \frac{\mathrm{d}^{2} N}{\mathrm{~d} \chi \mathrm{d} t}(\eta, \chi) \mathrm{d} \chi,
\end{aligned}
$$

where

$$
\frac{\mathrm{d}^{2} N}{\mathrm{~d} \chi \mathrm{d} t}(\eta, \chi)=\frac{\sqrt{3}}{2 \pi \tau_{\mathrm{C}}} \alpha_{\mathrm{f}} \frac{\eta}{\gamma} \frac{F(\eta, \chi)}{\chi}
$$

is the differential rate of production of photons of parameter $\chi$ by an electron of parameter $\eta$ and $F(\eta, \chi)$ is the quantum synchrotron emissivity, expressed in the weak, quasi-stationary field approximation [14]. An expression for $F(\eta, \chi)$ is given in Appendix A of [9]. The Compton time is $\tau_{\mathrm{C}}=\lambda_{\mathrm{C}} / c$, where $\lambda_{\mathrm{C}}=\hbar / m_{\mathrm{e}} c$ is the Compton wavelength, and $\alpha_{\mathrm{f}}$ is the fine structure constant.

When $\tau_{\mathrm{e}}=\tau_{\mathrm{e}}^{(\mathrm{f})}$ is reached, a photon is emitted, and its energy is randomly assigned as follows. First, the parameter $\chi^{(\mathrm{f})}$ is found from the relation

$$
\xi=\frac{\int_{0}^{\chi^{(\mathrm{f})}} \frac{\mathrm{d}^{2} N}{\mathrm{~d}_{\chi} \mathrm{d} t} \mathrm{~d} \chi}{\int_{0}^{\eta / 2} \frac{\mathrm{d}^{2} N}{\mathrm{~d}_{\chi} \mathrm{d} t} \mathrm{~d} \chi} \quad\left(=\frac{\int_{0}^{\chi^{(\mathrm{f})}} \frac{F(\eta, \chi)}{\chi} \mathrm{d} \chi}{\int_{0}^{\eta / 2} \frac{F(\eta, \chi)}{\chi} \mathrm{d} \chi}\right),
$$

where $\xi$ is a uniformly distributed random number in $[0,1]$, and the right hand side is a monotonic, increasing function of $\chi^{(\mathrm{f})}$, which is tabulated in both the $\chi^{(\mathrm{f})}$ and $\eta$ directions. The variables $\chi^{(\mathrm{f})}$ and $\eta$, in equation (7), depend on the electromagnetic field at the point of photon emission. Then, assuming that the photon is emitted parallel to the electron momentum, its energy $h v$, is determined by the relation

$$
h v=\frac{2 m_{\mathrm{e}} c^{2} \chi^{(\mathrm{f})} \gamma}{\eta}
$$


which follows from the definitions of $\eta$ and $\chi$ and the assumption that the electron is relativistic, $\gamma \gg 1$. For computational efficiency, we ignore the rare photons emitted in the low energy part of the spectrum, $\chi^{(\mathrm{f})}<\chi_{\min }^{(\mathrm{f})}$, where $\chi_{\text {min }}^{(\mathrm{f})}$ is chosen such that the neglected photons carry only $10^{-9}$ of the total energy and are incapable of producing pairs. The computational procedure was successfully tested by its ability to reproduce the correct synchrotron spectrum. This is shown in Fig.1, for a large number $\left(10^{6}\right)$ of emitted photons in a regime where $\eta=0.1$. A $\chi^{(f)}$ parameter, which represents the photon energy, is assigned to each photon at the emission point, according to the sampling relation (7). The occurrence (in percent of the total number of the $10^{6}$ emission events) of emissions in each energy interval gives a distribution along the $\chi^{(f)}$ axis, that scales with the quantum synchrotron emissivity $F\left(\eta, \chi^{(f)}\right)$.

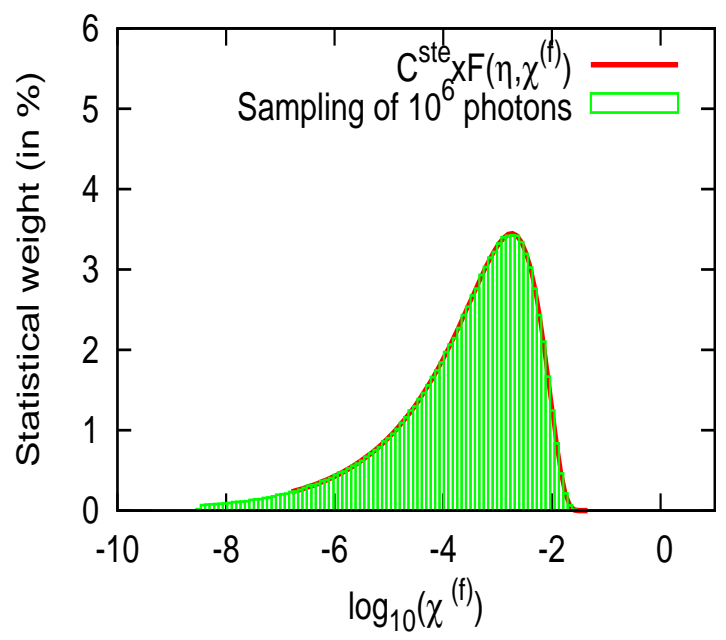

Figure 1. Comparison between the distribution in $\chi^{(f)}$ resulting from the sampling relation (7) and the quantum synchrotron emissivity $F\left(\eta, \chi^{(f)}\right)$.

After the electron has emitted a photon, which is assumed to occur instantaneously, it continues its trajectory beginning at the same point but with a different energy $\gamma^{(\mathrm{f})} m c^{2}$ and momentum $\boldsymbol{p}^{(\mathrm{f})}$. To find $\boldsymbol{p}^{(\mathrm{f})}$, we again use the approximation that the photon is emitted parallel to the pre-emission electron momentum, so that, by conservation of momentum,

$$
\boldsymbol{p}^{(\mathrm{f})}=\left(1-\frac{h v}{c p}\right) \boldsymbol{p} .
$$

In reality, the photon may be emitted over a cone of opening angle approximately $1 / \gamma$, but we neglect this small angular spread here. Equation (9) implies that a small amount of energy is extracted from the laser fields during photon emission, which we also neglect. This neglect is unimportant in the present test-particle model in which the laser fields are prescribed, since the fraction of the electron energy transferred to the laser field is small $\sim 1 /\left(\gamma \gamma^{(\mathrm{f})}\right)$, as shown in Appendix A. However this point may need more careful treatment in a more complete dynamic treatment of the development of an electron-positron cascade and its inclusion in a particle-in-cell code [15, 16]. 


\section{Straggling effects}

As in [9], we adopt the configuration of two counter-streaming, circularly polarized laser beams, which can qualitatively be considered equivalent to the interaction of an incident and reflected beam from a solid [8]. We restrict the treatment to pair creation by the two-step process described in section 2, Pairs can also be produced directly by an electron interacting with the laser field, without the need to produce an intermediate real photon. This process is analogous to trident pair production by an electron in the Coulomb field of a nucleus. However, although this direct, single-step process may be important at lower laser intensity, it is dominated by the indirect two-step process at laser intensities above $10^{23} \mathrm{Wcm}^{-2}$, and we do not include it in the results presented here.

In the two-step process, the emitted gamma-rays travel through the plasma and may produce pairs according to the cross-section which depends on $\chi$. In many ways, the process is comparable with that of gamma-ray production by electrons. The photon trajectory could be followed through the electromagnetic fields and the optical depth integrated as for electrons. However, in order to make a direct comparison with the rate computed assuming continuous losses, we here follow the much simpler approximate approach that was used in [9]. The probability of pair production is calculated for a gamma-ray with $\chi=\chi^{(\mathrm{f})}$, that propagates through electromagnetic fields that are assumed to equal those at the point where the gammaray was emitted. We follow [9] and make the assumption that conversion into pairs can occur until the photon has propagated a distance of one laser wavelength. Here the approximation is that the beams have a transverse structure with cylindrical geometry, whose radius is one wavelength, representative of beams which are tightly focussed at the diffraction limit to achieve maximum laser intensity. The real photons only produce pairs while they propagate through the region of large laser field. In contrast to electrons, whose trajectory is determined by the electromagnetic fields, the photons are undeflected. This means that all along its path through the laser fields, the value of the parameter $\chi$ remains unchanged at $\chi=\chi^{(\mathrm{f})}$. Further details can be found in section 3.3 of [9]. The differential pair production rate via real photons is the product of the differential rate for real photon emission, $\frac{\mathrm{d}^{2} N}{\mathrm{~d} \chi \mathrm{d} t}(\eta, \chi)$, and an effective conversion probability into pairs, $\left(1-\exp \left(-\left\langle\tau_{\mathrm{p}}\right\rangle\right)\right)$, where $\left\langle\tau_{\mathrm{p}}\right\rangle$ is an effective optical depth to pair conversion. The rate of pair production via real photons per electron is then addressed with the same rate equation as in [9]

$$
\frac{\mathrm{d} N_{\text {real }}}{\mathrm{dt}}=\int_{0}^{\eta / 2} \mathrm{~d} \chi \frac{\mathrm{d}^{2} N}{\mathrm{~d} \chi \mathrm{d} t}(\eta, \chi)\left(1-\exp \left(-\left\langle\tau_{\mathrm{p}}\right\rangle\right)\right) .
$$

\subsection{Cumulative effects of the straggling at a $B=0$ node}

In this section, we select a particular trajectory at a $\boldsymbol{B}=0$ node (zero magnetic field) of an infinite standing wave generated by two counter-propagating, circularly polarized laser beams with the same sense of rotation of the fields. Throughout, distances and times are scaled with the laser wavelength and laser period, respectively. This configuration has been extensively analysed in [8, 9] in the case of continuous radiation losses where it was shown that the 
electron settles into a circular orbit, which is favourable for pair production. Here we show how discontinuous trajectories increase pair production.

The two beams counter-propagate along the $z$-axis, each with an intensity $I_{24} \times$ $10^{24} \mathrm{~W} \mathrm{~cm}^{-2}$, with $I_{24}=0.3$. The electron is initially located at a $\boldsymbol{B}=0$ node in the standing wave formed by the counter-propagating beams. Its initial Lorentz factor is $\gamma=10$, with its momentum oriented along the positive $y$-axis. The electron motion is tracked over seven laser periods during which it remains at the magnetic node. In Fig. 2(a), the discontinuous Monte Carlo calculations lead to the straggling of the Lorentz factor for the particle (green curve). This is compared with the Lorentz factor of a particle that undergoes continuous radiation loss, computed as in [9] (red curve) for the same initial conditions. A feature of the straggling in this regime is a $\sim 30 \%$ fluctuation about the Lorentz factor derived from the continuous description. The particle motion, as shown in Fig. 2(b) is characterized by excursions to higher and lower $\eta$ values than those reached by an electron undergoing continuous losses. The discrepancies in the particle $y$ position, between the two descriptions, is also shown in Fig. 2(b). Finally, the comparison of Fig. 3, and Fig. 2(b), shows that the high $\eta$ excursions are very effective at producing pairs. Moreover, the accumulation of pairs, produced by the high $\eta$ excursions, leads to an enhancement of the overall pair production, in this example by about $40 \%$ after only seven laser periods. At this time, the average number of pairs produces by an electron reaches unity, which can be considered an approximate threshold for the initiation of an electron-positron avalanche.

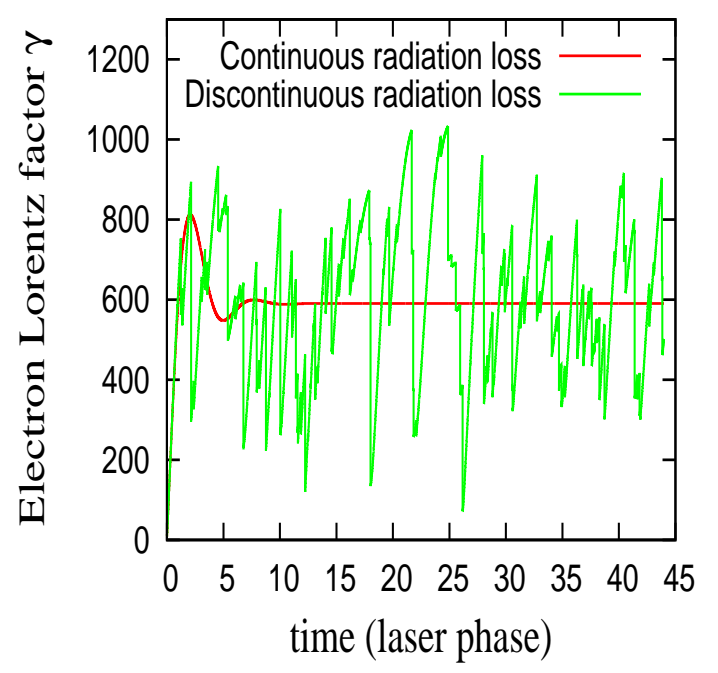

(a)

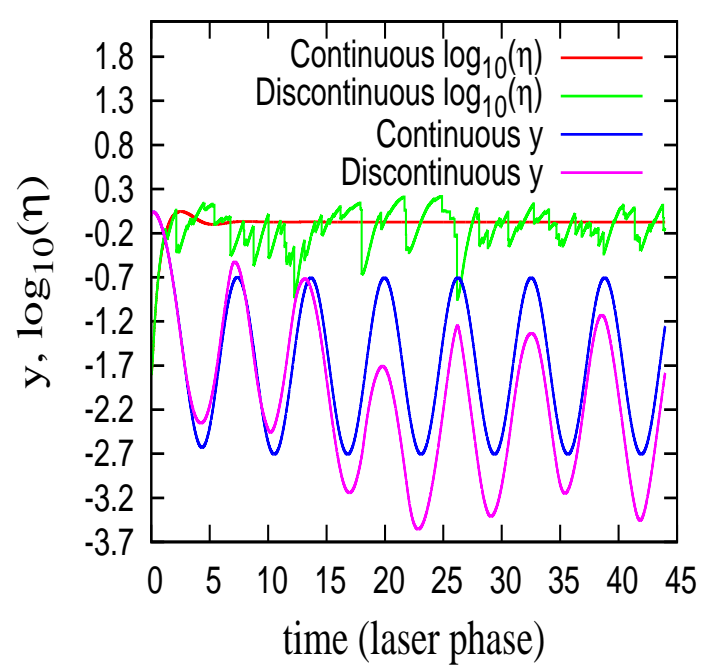

(b)

Figure 2. Case of an electron at a $\boldsymbol{B}=0$ node of two very long, counter-propagating, circularly polarized pulses. The trajectories found with continuous and discontinuous lossdescriptions are compared in terms of (a) Lorentz factor, (b) $\eta$-parameter \& spatial coordinate $y$. 


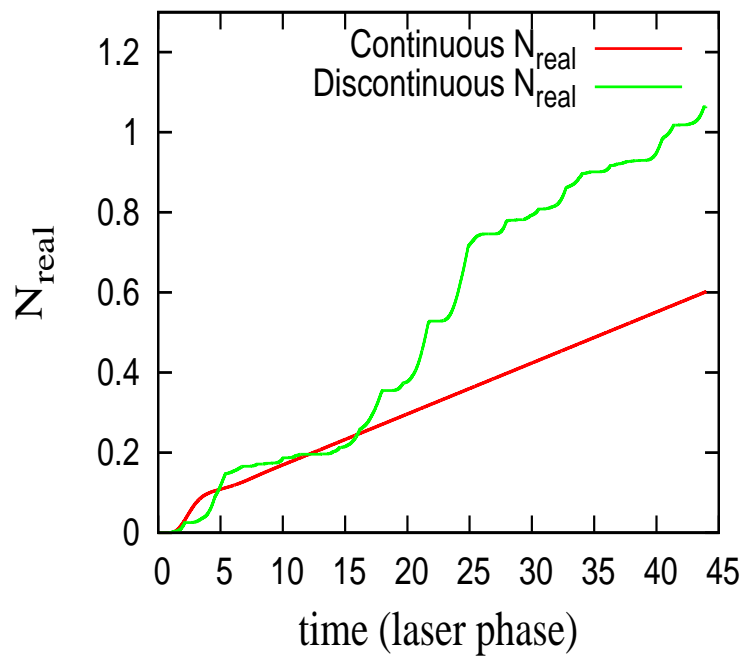

Figure 3. Number of pairs produced against time, by the continuous-loss trajectory (red curve) and the discontinuous one (green curve).

\subsection{Convergence properties of discontinuous trajectories, off- $B=0$ nodes}

In [9], the authors showed that the electron trajectory at a $\boldsymbol{B}=0$ node is unstable. Small displacements from the node grow, causing the electron to migrate towards the stable $\boldsymbol{E}=0$ nodes. When the electron reaches an $\boldsymbol{E}=0$ node, its oscillatory momentum damps towards zero and pair production ceases. Here, we show that in the more realistic Monte Carlo description, the migration progresses less smoothly, and, on average, the electrons tend to take longer to reach to the $\boldsymbol{E}=0$ node. This increases the number of pairs produced, since, on average, electrons emit high energy photons for a longer period of time.

We consider the same configuration and initial conditions used in section 3.1, with $I_{24}=0.3$, except that the initial electron position is now $z=0.1$ instead of $z=0$, which is the location of the $\boldsymbol{B}=0$ node. Then the motion of a set of $10^{4}$ electrons is followed until the time $t_{\max }=20$. Because of random fluctuations, each of the $10^{4}$ trajectories is different, resulting in a different number of pairs finally produced. In Fig. 4(a), the z-position for two sample trajectories is tracked, and compared with the $z$-position of an equivalent electron starting from the same point but subject to a continuous radiation-reaction force. Two differing kinds of behaviour are highlighted. In one case (the blue curve), an electron trajectory described by discontinuous radiation losses reaches the stable $\boldsymbol{E}=0$ node earlier than the trajectory described by continuous radiation losses (red curve). In another discontinuous-loss case (green curve), the electron takes longer to converge on the stable $\boldsymbol{E}=0$ node. The majority of electrons belong to this latter class. This is demonstrated quantitatively in Fig. 4(b), where the red curve shows the evolution of the square of the distance to the $\boldsymbol{E}=0$ node at $z=\pi / 2$, averaged over $10^{3}$ discontinuous trajectories, i.e., $\left\langle(z-\pi / 2)^{2}\right\rangle$. For comparison, the green curve shows the case of continuous radiation loss. The longer migration time, together with the excursions to large $\eta$ discussed above, combine to enhance pair production 
even further. The overall increase in pair production is shown in Fig. 4(c) which presents the distribution function for pair production compiled from $10^{4}$ trajectories. The $N_{\text {real }}$ axis is divided into 100 equally spaced intervals in the logarithmic range $\log _{10}\left(N_{\text {real }}\right) \in[-5,1]$. All the electrons are identically initialized at $z=0.1$, with $I_{24}=0.3$ (red bars). We find that the spectrum is substantially shifted upwards with respect to the continuous value (green line), $\log _{10}\left(N_{\text {real }}\right) \simeq-1.01$, obtained with continuous radiation loss. The figure shows that $77.5 \%$ of the particles produce more pairs than if their trajectory was computed in a continuous manner. A small number of the sampled trajectories come close to producing one pair per electron $\left(\log _{10}\left(N_{\text {real }}\right) \simeq 0\right)$.

For the same configuration and initial conditions, we now repeat the calculation at a lower laser intensity, $I_{24}=0.1$. The results are presented in Fig. 5(a), where the $N_{\text {real }}$ axis is again divided into 100 equally spaced intervals, this time in the logarithmic range $\log _{10}\left(N_{\text {real }}\right) \in[-6,0]$. We find that the distribution is again shifted upwards with respect to the value $\log _{10}\left(N_{\text {real }}\right) \simeq-4.19$ obtained with the continuous radiation loss. Electron energy straggling and longer migration times prove to have a significant impact on the number of pairs produced. For the top $1 \%$ of the trajectories, one order of magnitude more pairs are produced than are predicted using continuous radiation losses.

In contrast, at higher laser intensities the spread of the distribution is quite narrow, and is centred close to the continuous value. This is illustrated in Fig. 5(b), where we present results for $I_{24}=1$. In this figure, the $N_{\text {real }}$ axis is divided into 100 equally spaced intervals in the logarithmic range $\log _{10}\left(N_{\text {real }}\right) \in[-2,4]$. The location of the peak in the spectrum agrees well with the number of pairs computed with a continuous semi-classical description $\log _{10}\left(N_{\text {real }}\right) \simeq 0.55$. At high laser intensity, straggling has less effect, because the process is above threshold and less dependent on statistically rare excursions to large $\eta$.

In order to obtain a more straightforward figure of merit, in terms of produced pairs, we compute an effective value for the number of pairs produced per electron whose motion is followed:

$$
\left\langle N_{\text {real }}\right\rangle=\sum_{i \in I} N_{\text {real }}(i) P(i)
$$

where $I$ stands for the complete set of equally spaced intervals that discretize the $N_{\text {real }}$ axis. The index $i$ refers to one of these intervals, in which the probability is $P(i)$. This procedure amounts to summing over histograms such as those in Fig. 4(c), 5(a) \& 5(b), for the total expected number of pairs produced per electron starting from an initial position $z=0.1$.

For a range of laser intensities, Fig. 6(a) shows a comparison between the number of pairs produced per electron undergoing discontinuous losses (computed using a Monte Carlo approach), with the number found for continuous losses. For low laser energy, $I_{24}=0.1$, the number of pairs $\left\langle N_{\text {real }}\right\rangle$ associated with discontinuous-loss trajectories is about 5 times larger than the number associated with continuous-loss trajectories. This is due to the importance of stragglers in this regime, and has a strong dependence on the $\eta$-parameter. Because of the large range in $N_{\text {real }}$ (eight orders of magnitude) this effect is more easily seen in Fig 6(b), which plots the ratio between these two numbers, showing that $\left\langle N_{\text {real }}\right\rangle / N_{\text {real }}$ shrinks as the laser intensity increases, until it becomes very close to unity, at $I_{24}=1$. The strength of the 


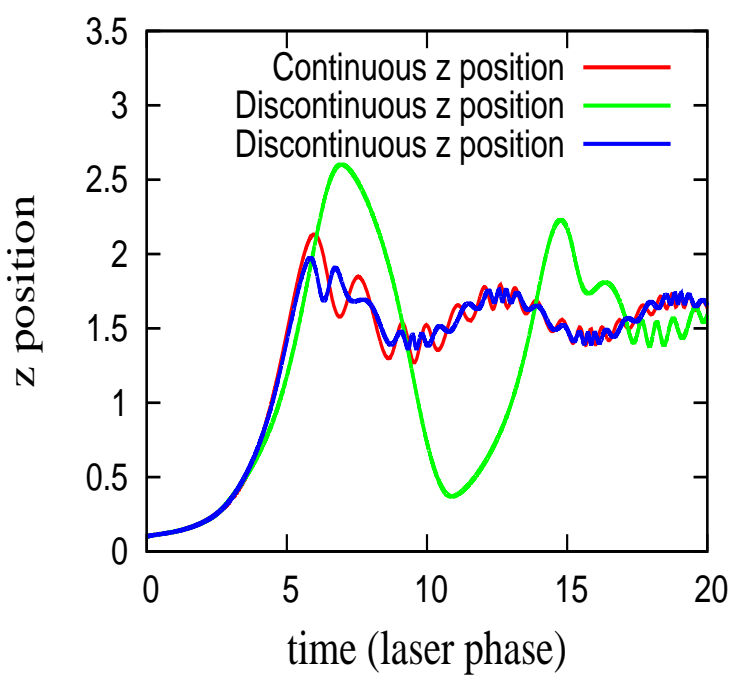

(a)

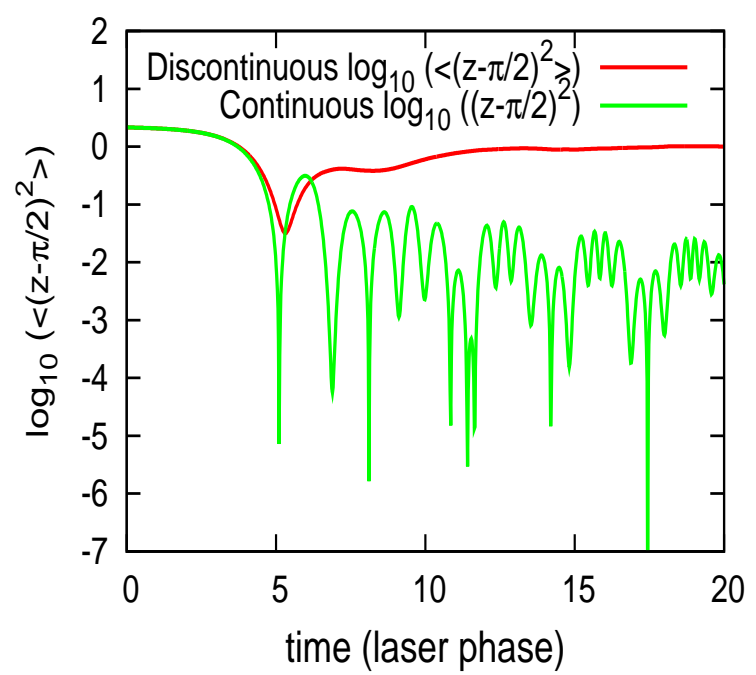

(b)

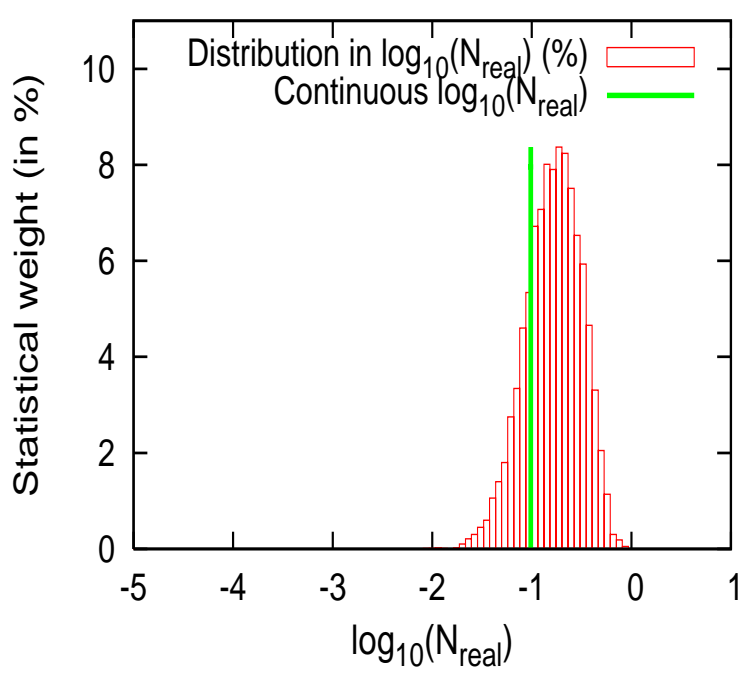

(c)

Figure 4. The discontinuous and continuous descriptions are compared at a laser intensity $I_{24}=0.3$, for initialization at $z=0.1$, away from the $\boldsymbol{B}=0$ node (located at $z=0$ ). In (a) $z$ is shown as a function of time, in (b) the square of the distance to the stable $\boldsymbol{E}=0$ node (located at $z=\pi / 2$ ), and in (c) the statistical distribution of the number of pairs $N_{\text {real }}$ produced by a trajectory.

straggling process is found to be very sensitive to the laser intensity for about $I_{24}<0.4$.

\section{Conclusion}

In this paper, we report on calculations of the number of electron positron pairs produced as an electron moves in the field of a laser with intensity in the range range $10^{23}-10^{24} \mathrm{~W} \mathrm{~cm}^{-2}$. In this regime, the dominant route to pair production involves the emission of gamma-ray photons via synchrotron radiation, and their further conversion into electron-positron pairs 


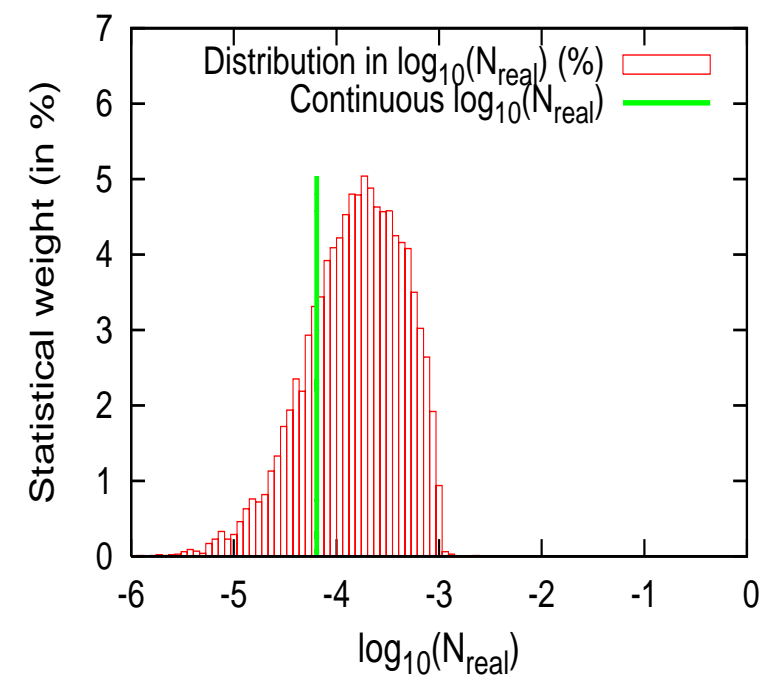

(a)

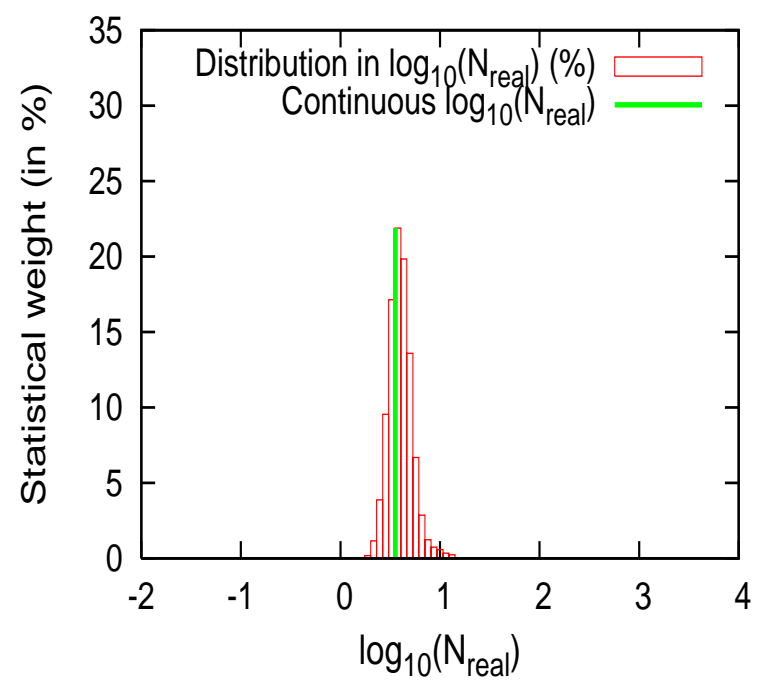

(b)

Figure 5. The statistical distribution (red bars) of a set of $10^{4}$ electrons identically initialized at $z=0.1$ (close to the $B=0$ node at $z=0$ ) for (a) $I_{24}=0.1$, and (b) $I_{24}=1$. The number of pairs produced by an electron undergoing continuous losses is shown for comparison (green lines).

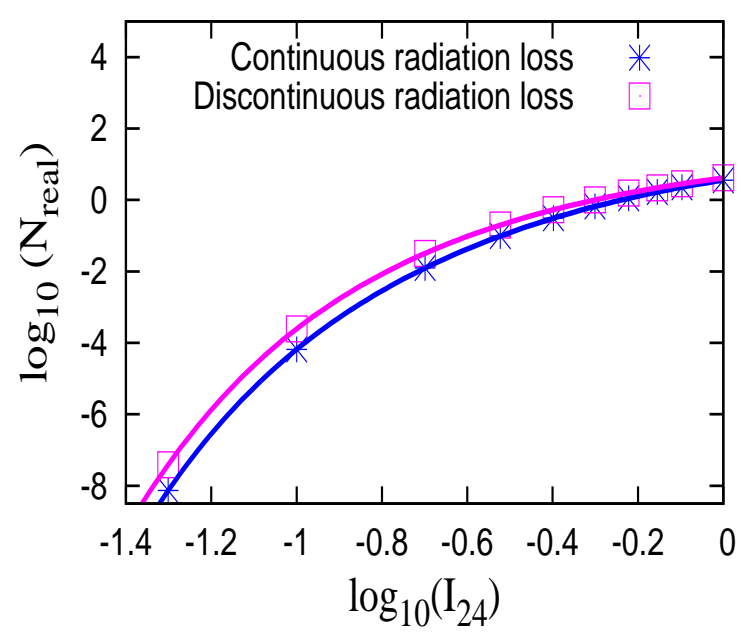

(a)

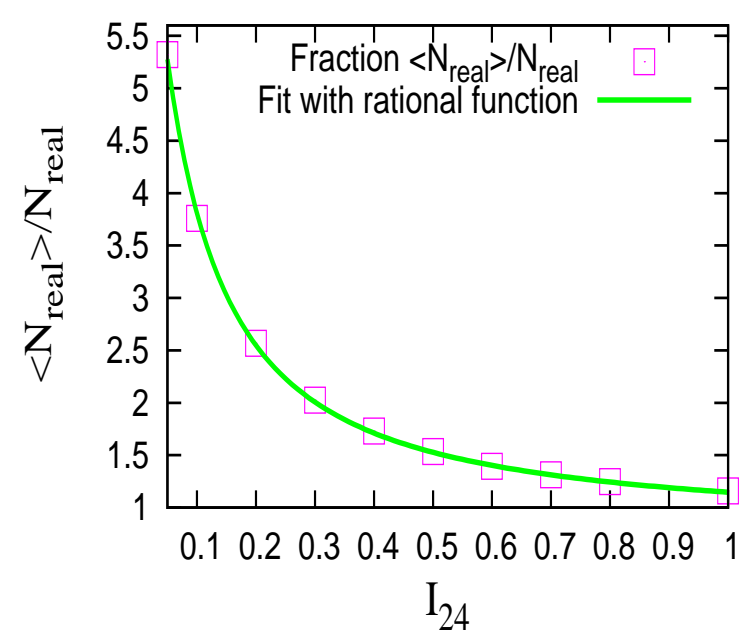

(b)

Figure 6. The continuous- and discontinuous-loss descriptions are compared in terms of the number of pairs produced, with respect to the laser intensity parameter $I_{24}$, for two counterstreaming, circularly polarized pulses, the particle being initialized at $z=0.1$, close to the $\boldsymbol{B}=0$ node at $z=0$. The curves are fits with rational functions. 
[8, 9]. The new aspect of our treatment is that we take account of the stochastic nature of gamma-ray production, which leads to discontinuous quantum jumps in the electron energy; using a Monte-Carlo algorithm we follow the evolution of a large number of stochastic trajectories that result from each individual set of initial conditions.

Pair production has been shown to be important in configurations with counterpropagating laser beams, that can be generalized to apply to laser-solid interactions [8, 9]. However, these authors used a continuous approximation for the radiation reaction force, and examined, therefore, one classical trajectory for each set of initial conditions. In order to make a direct comparison with our calculations, we investigate a specific, highly idealized configuration of the laser fields that was also used as a test-case by [8, 9]: that of two long, counterpropagating, circularly polarized laser pulses.

Discontinuous, stochastic jumps in the electron energy give rise to a spread in energy as a function of time known as straggling. Our main result is that straggling increases the number of pairs produced for given initial conditions, and for a given laser intensity. This behaviour is more pronounced for laser intensities less than $0.4 \times 10^{24} \mathrm{Wcm}^{-2}$ where the transition probabilities are strongly dependent on $\eta$ and $\chi$; in this range we observe up to a fivefold increase in the number of pairs produced. Straggling effects are less important at higher intensities, where most gamma-rays are above threshold for pair production and the number of electron-positron pairs produced is large.

Two effects contribute to the increase in pair production due to straggling. Firstly, the value of the electron's $\eta$-parameter, which controls the energy of the emitted gamma-ray, varies considerably about its mean. Occasional excursions to large $\eta$ result in the emission of higher energy gamma-rays with a correspondingly larger probability of producing pairs. Secondly, straggling increases, on average, the time taken for electrons to congregate in a region where few pairs can be created (a node with $\boldsymbol{E}=0$ in our calculations), and this extends the time during which high-energy photons are emitted and pairs produced. We conclude that straggling lowers the laser intensity required for significant pair production.

In realistic situations, when large numbers of pairs are produced, their mutual interactions as well as their back-reaction on the accelerating fields must be taken into account. In an astrophysical setting, [16] has addressed this problem by combining a Monte-Carlo algorithm with a PIC-code. We envisage that the Monte Carlo algorithm we present will serve as a basis for a coupled Monte Carlo-PIC algorithm aimed at modelling the initiation of prolific pair-plasma creation in intense laser fields.

\section{Acknowledgments}

We thank the UK Engineering and Physical Sciences Research Council for support under grant number EP/G055165/1, entitled 'Multi-scale simulation of intense laser plasma interactions'. 


\section{References}

[1] Mourou G A, Labaune C L, Dunne M, Naumova N, and Tikhonchuk V T 2007 Relativistic laser-matter interaction: from attosecond pulse generation to fast ignition Plasma Physics and Controlled Fusion 49 B667-B675

[2] http://www.eli-laser.eu

[3] Chekhov O, Collier J, Clark R J, Hernandez-Gomez C, Lyachev A, Matousek P, Musgrave I O, Neely D, Norreys P A, Ross I, Tang Y, Winstone T B, Wyborn B E 2009 The 10 PW OPCPA Vulcan Laser Upgrade IEEE 978-1-4244-4080-1

[4] Hernandez-Gomez C, Blake S P,Chekhov O, Clarke R J, Dunne A M, Galimberti M, Hancock S, Holligan P, Lyachev A, Matousek P, Musgrave I O, Neely D, Norreys P A, Ross I, Tang Y, Winstone T B, Wyborn B E, and Collier J 2008-2009 The Vulcan 10 PW project CLF annual report, http://www.clf.rl.ac.uk

[5] Salamin Y I, Hu S X, Hatsagortsyan K Z, and Keitel C H 2006 Relativistic high-power laser matter interactions, Phys. Rep. 427, 41-155

[6] Müller C, Hatsagortsyan K Z, Ruf M, Müller S J, Hetzheim H G, Kohler M C and Keitel C H 2009 Relativistic nonperturbative above-threshold phenomena in strong laser fields, Laser Physics 19, 17431752

[7] di Piazza A, Hatsagortsyan K Z and Keitel C H 2009 Strong Signatures of Radiation Reaction below the Radiation-Dominated Regime, Phys. Rev. Lett. 102, 254802

[8] Bell A R and Kirk J G 2008 Possibility of Prolific Pair production with High-Power Lasers, Phys. Rev. Lett. 101200403

[9] Kirk J G, Bell A R and Arka I 2009 Pair production in counter-propagating laser beams Plasma Physics and Controlled Fusion 51085008

[10] Fedotov A M, Narozhny N B, Mourou G, and Korn G 2010 Limitations on the attainable intensity of high power lasers arXiv: 1004.5398

[11] Sokolov I V, Naumova N M, Nees J A, Mourou G A, and Yanovsky V P 2009 Dynamics of emitting electrons in strong laser fields Phys. Plasma 16093115

[12] Shen C S and White D 1972 Energy Straggling and Radiation Reaction for Magnetic Bremsstrahlung Physical Review Letters 287

[13] Shen C S 1972 Magnetic Bremsstrahlung in an Intense Magnetic field Physical Review D 610

[14] Erber T 1966 High-energy electromagnetic conversion processes in intense magnetic fields Rev. Mod. Phys. 38 626-59

[15] Sokolov I V, Nees J A, Yanovsky V P, Naumova N M, Mourou G A 2010 Emission and its back-reaction accompanying electron motion in relativistically strong and QED-strong pulsed laser fields Phys. Rev. E 81036412

[16] Timokhin A N 2009 Self-consistent modeling of pair cascades in the polar cap of a pulsar Fermi Symposium, Washington D.C., Nov. 2-5 


\section{Appendix A. Estimation for the energy transferred to the laser field by a single particle}

From the momentum change expression at the photon emission time $\mathbf{p}^{(\mathrm{f})}=\mathbf{p}-\hbar \mathbf{k}$, we estimate the error, in the Monte Carlo algorithm, related to the energy transferred to the laser field by a single particle. The expression for the relative error reads

$$
\frac{\Delta \gamma}{\gamma} \equiv \frac{1}{\gamma}\left|\gamma^{(\mathrm{f})}-\gamma+h v /\left(m_{\mathrm{e}} c^{2}\right)\right|
$$

In the limit where the $1 / \gamma$ and $1 / \gamma^{(\mathrm{f})}$ parameters are small, it can be approximated as

$$
\frac{\Delta \gamma}{\gamma} \simeq \frac{1}{2} \frac{1}{\gamma}\left(\frac{1}{\gamma^{(\mathrm{f})}}-\frac{1}{\gamma}\right),
$$

which is of the order $1 /\left(\gamma \gamma^{(\mathrm{f})}\right)$. 